\title{
Retrospectiva agroecológica a la interacción en- tre sistemas agrícolas tradicionales y modernos.
}

\author{
René Montalba Navarro ${ }^{1}$
}

\begin{abstract}
Resumen
En este trabajo se realiza un acercamiento desde la agroecología crítica a los orígenes y la expansión de la "agricultura moderna" y a su extensión hacia los sistemas agrícolas tradicionales del tercer mundo. Así también, se analizan los efectos de esta transformación y de la adopción de las prácticas genéricas de la agricultura moderna en los sistemas agrícolas familiares, principalmente campesinos e indígenas.

Los antecedentes históricos recopilados ponen de manifiesto que este proceso ha sido ampliamente desfavorable para los sistemas familiares, particularmente en aspectos económicoproductivos, ambientales y sociales; encontrando el origen de gran parte de sus problemas actuales en la expansión de la "nueva agricultura" y en el modelo de desarrollo que hay tras ella.

Con el respaldo de argumentos de tipo empírico y teóricos actuales, en este trabajo se concluye que, al igual que lo que ha ocurrido en otros lugares del planeta, la "modernización" de los sistemas agrícola familiares, con base tradicional, no dará solución al estado de pobreza material en la cual se encuentran campesinos e indígenas de los países del tercer mundo, sino que por el contrario producirá otros tipos de pobreza y problemas de diversa índole, muchos de ellos con efectos inciertos.
\end{abstract}

\begin{abstract}
In this work a critical agroecological approach is carried out in order to evaluate the origins of "modern agriculture" and its expansion toward the traditional agricultural systems of the third world. The effects of this transformation (adoption of the generic practices from "modern agriculture" into traditional agricultural systems) are analyzed. The empirical and theoretical data analysed in this paper, led us to conclud that "modernization" of the traditional agricultural systems will not
\end{abstract}

give solution to the material poverty of peasants and native populations of the third world, but on the contrary could produce another kind of problems, many of them with uncertain effects.

\section{Introducción}

$\mathrm{G}$ ran parte de la población rural indígena de nuestro país, al igual que la de muchos de los países del "tercer mundo", se encuentra en estado de pobreza o de extrema pobreza (material), lo cual implica que no pueden satisfacer sus necesidades básicas y muchas veces ni siquiera sus requerimientos nutricionales. Dada esta difícil situación, ha sido planteado que la solución a estos problemas pasa por lograr un aumento de la productividad y "calidad" de la producción mediante la "modernización" de sus sistemas económico-productivos. Generalmente esta "modernización" se refiere al reemplazo de sus prácticas productivas por aquellas que se utilizan en los sistemas agrícolas más tecnificados y el cambio de orientación económica, desde una economía de características "campesinas" a otra más liberal y orientada al mercado.

Por otro lado, estamos quienes consideramos que la adopción de las prácticas y la lógica productiva de la "agricultura moderna", no solo no han dado ni darán solución a la pobreza y el hambre en los sectores campesinos e indígena (rural) de los países del tercer mundo, sino que en la mayoría de los casos en los cuales se realice esta transformación, los problemas se agudizarán y serán producidos otros nuevos, tanto de carácter social y económico como ambiental y cultural.

Como una forma de argumentar estos planteamientos, en el presente trabajo se recopilan los antecedentes históricos tanto del origen y desarrollo de la "agricultura moderna", comr extensión a los países del tercer mundo reemplazando los sistemas tradicionales, y se organizan e interpretan desde las perspectivas propi:stas por la historia ecológica y la ecología política. También son analizados los principales efectos que la trans-

1 Facultad de Ciencias Agropecuarias y Forestales, Instituto del Medio Ambiente, Universidad de la Frontera, Temuco, Chile. 
formación de estos sistemas ha implicado, prestando especial atención a los sistemas familiares y comunitarios de los países del "tercer mundo", principales objetos de estudio de la agroecología y la economía ecológica. Cabe destacar que, pese a la directa relación entre estos, no se considerarán todos los efectos relacionados con el modelo de desarrollo al cual esta adscrita la "Revolución Verde" sino que solo los más directamente relacionados con la transformación tecnológica que esta implica.

\section{Sobre el origen y expansión de la "agri- cultura moderna"}

Bajo el concepto de "agricultura tradicional" (o pre-científica) podemos agrupar a aquellos tipos de agricultura o sistemas agrícolas que tanto en su desarrollo como en su funcionamiento no responden a la lógica científica y económico-mercantilista de los sistemas "modernos", sino que más bien obedecen a concepciones de hombre y naturaleza diversos, que se han originado y desarrollado a través del tiempo mediante múltiples relaciones entre los diferentes grupos y el medio el cual habitan. De este modo, en la agricultura tradicional, el desarrollo de sus sistemas es el resultado de una coevolución, en el sentido de evolución integrada, entre cultura y medio ambiente (Sevilla Guzmán y González de Molina, 1990). Esta se ha beneficiado de siglos de evolución cultural y biológica, mediante lo cual se ha adaptado a las condiciones locales. Así, los agricultores han creado y/o heredado sistemas complejos de agricultura que, durante siglos, los han ayudado a satisfacer sus necesidades de subsistencia, incluso bajo condiciones ambientales muy adversas (Altieri, 1999). Este proceso ha contribuido al origen de diversas culturas en interacción con medio ambientes también diversos, en los cuales, y por medio de la estrecha relación entre los agricultores y la naturaleza, el hombre ha desarrollado un basto conocimiento de las distintas especies naturales y sus ecosistemas, domesticando una gran diversidad de especies y variedades de estas.

Los sistemas agrarios tradicionales han debido adaptarse a los límites marcados por las características de suelo y clima de cada zona, adaptación que se apoyó tanto en la selección de los cultivos y aprovechamientos, como en las prácticas agrarias aplicadas. Tanto en el marco de plantación, como las rotaciones de cultivo o la trashumancia, estos se ajustaban a las limitaciones edafoclimáticas de su territorio (Naredo, 1996). Debido a lo anterior, en general los sistemas tradicionales poseían una alta estabilidad y capacidad de recobrarse de perturbaciones ambientales (Altieri, 1999). Sin embargo, se debe mencionar también el hecho de que los rendimientos individuales (sin considerar los policultivos) de los principales cultivos eran más bien modestos.

Pese a que los precedentes más directos de la transformación de los sistemas agrícolas, mediante la aplicación de la ciencia y la tecnología, los podemos encontrar en la segunda mitad del siglo XIX, los mayores avances y cambios se lograron con posterioridad a la Segunda Guerra Mundial, al converger un nuevo conjunto de innovaciones científico-tecnológico con la consolidación de ideologías político-económicas (Pujol, 1998). Esto permitió la introducción a la agricultura de los "esquemas racionales" que el negocio de la industria había seguido (Toledo, 1993; Sevilla Guzmán y Lopez Calvo, 1994), teniendo como uno de sus efectos un gran incremento en la producción y rendimiento de cultivos como el arroz, maíz y el trigo en los países desarrollados de Europa y Norteamérica. Como resultado, se creó un nuevo tipo de agricultura (que se ha denominado "de altos inputs", "agricultura químico-genética" o "agricultura científica", entre otros) que reemplazaría a los sistemas más tradicionales de estos países (Chrispeels y Sadava, 1994).

Tras la apropiación por parte de Trumann del concepto de "desarrollo" y derivándose de este los de "subdesarrollo" y "países subdesarrollados", la atención se dirigió cada vez más hacia los problemas de largo plazo relacionados con la "ayuda" para el "desarrollo económico" de áreas "subdesarrolladas" (Escobar, 1996). En esta dirección, y considerando que el hambre crónica bajo la cual vivían muchos de los habitantes de los países "sub desarrollados" se debía a la ineficiencia y baja productividad de sus sistemas agrícolas (con fuerte base tradicional), se comienza a plantear que la solución al problema del hambre se encontraba en traspasar a estas zonas los adelantos en el campo de la agricultura conseguidos en los países industrializados, logrando con esto su suficiencia alimentaria. Cabe destacar que tras estos objetivos altruistas se enmascaraba una estrategia de expansión de la influencia estadounidense sobre los países del tercer mundo, intereses de la industria petrolera, intereses de la industria de insumos agropecuarios y otros aún menos nobles (Ross, 1998).

Una de las principales dificultades de este traspaso tecnológico se encontraba en el hecho de que la "agricultura moderna" había sido desarrollada en zonas con clima templado y los lugares en los cuales se requería adaptar correspondía más bien a climas tropicales o sub-tropicales. Dado lo anterior, y debido a que las variedades positivamente sensibles al uso de fertilizantes solubles son el eje central de estos sistemas agrícolas, a lo primero que se debía apostar era a desarrollar variedades de alto rendimiento adaptadas a estas condiciones. Los primeros en embarcarse en esta "apuesta" fueron fundaciones como Rockefeller y Ford, mediante la financiación de inicis. - . tras las cuales comienza a gestarse la renombrada "Revolucion Verde". Los pioneros de esta tarea revolucionaria, que empezó en Méjico, fueron cuatro científicos norteamericanv (financiados por la Fundación Rockefeller), quienes pudieron desarrollar variedades de trigo de alta productividad adaptadas a las condiciones edafoclimáticas distintas a las templadas. Su obra fue continuada por el Dr. Orville Vogel y el Dr. Norman Borlaug, quienes cruzaron con éxito variedades de trigo de alto rendimiento y enanas, adecuadas a las condiciones de una estación experimental de Méjico. Esta estación se convirtió posterior- 
mente en el Centro Internacional de Mejoramiento de Maíz y Trigo (CIMMYT). El éxito de las variedades de trigo de alta productividad animó a todo el mundo, de modo que en los años sesenta se creó el Instituto Internacional de Investigación del Arroz (IRRI, International Rice Research Institute) y tras este otros más relacionados con los principales cultivos económicos y alimenticios, los cuales se convirtieron en sinónimo de variedades de aita productividad (BALAI, 1987). Desde la creación de los Institutos Internacionales (financiados por países del primer mundo) y hasta avanzados los ochenta, estos se enfocaron en la propuesta que hasta entonces les había resultado exitosa, relacionada con la obtención de variedades altamente productivas de maíz y trigo. Esto significa que se concentraron en la utilización de cultivos puros (monocultivos) y la productividad de dichos cultivos mediante un paquete tecnológico, que incluía variedades mejoradas asociadas a tecnologías de modificación de ambiente (riego, fertilización, pesticidas, etc.).

En este período, los distintos gobiernos de los países "sub desarrollados" fueron convencidos de las bonanzas del nuevo sistema agrícola y seducidos por la promesa del "fin del hambre en el mundo". De este modo, financiados por préstamos del Banco Mundial y otros organismos financieros internacionales, se aplican ambiciosos programas de transferencia tecnológica orientados a mostrar a los agricultores la superioridad de las nuevas prácticas y sistemas agrícolas y entregar lo necesario para su adopción, lo cual en la mayoría de las ocasiones se tradujo en asistencia técnica y créditos (y/o subsidios) para el uso y compra de fertilizantes, pesticidas, maquinarias y semillas mejoradas. Esto se realizó muy masivamente y sin considerar la relatividad geográfica y cultural de las distintas zonas, además de los requerimientos constantes de las variedades y paquetes tecnológicos de este tipo.

\section{Efectos de la agricultura moderna en los sistemas agrícolas familiares del "tercer mundo"}

$\mathrm{Al}$ analizar los efectos de la agricultura "moderna" y de la adopción de esta en los sistemas familiares y comunitarios indígenas, resulta importante el destacar que estos no solo se producen por la incorporación de prácticas y la realización de cambios en los sistemas productivos propios. Al igual que la incorporación de las tecnologías del tipo "revolución verde" presenta una serie de efectos en estos sistemas, la aplicación masiva del modelo de desarrollo agrícola que hay tras ellas tiene efectos tanto o más importantes.

Al momento de ver los efectos de la incorporación de las prácticas modernas en los sistemas familiares del tercer mundo, debemos diferenciar por lo menos entre dos formas principales de ocurrencia: a) Con el apoyo de programas de transferencia tecnológica (gubernamentales y no gubernamentales);

b) Con muy escaso o sin apoyo de programas de transferencia tecnológica.

El primero de estos (a) se refiere a aquellas situaciones en las cuales la transformación productiva ha sido apoyada técnica y muchas veces monetariamente por programas estatales o de otra índole, los cuales permiten (o exigen) la adopción de todo el paquete tecnológico. A diferencia, la segunda (b) corresponde a los casos en los cuales algunas prácticas son incorporadas por imitación a los agricultores más tecnificados o por trasferencias puntuales con escaso seguimiento y también escaso o nulo apoyo financiero.

A la luz de las cifras resulta innegable el hecho de que la primera situación se ha dado más bien en el sector de la mediana a gran propiedad, donde este tipo de paquetes tecnológicos resul$\tan$ "viables" y a la vez "rentables". La gran mayoría de los sistemas agrícolas familiares, con características tradicionales, que ocupan terrenos marginales desde el punto de vista productivos y con extrema fragilidad desde el punto de vista ambiental, no se han "beneficiado" de este tipo de programas (Altierí, 1999; Rosset, 2001), y por tanto la adopción de las prácticas modernas se ha producido más bien de la segunda forma (b). De esta manera los agricultores implementan las prácticas que más les acomodan (por condiciones socio-culturales o características edafoclimáticas) o a las que tienen acceso.

Debido a que en los sistemas agrícolas tradicionales es una práctica común el intercambio de semillas y "experimentar" con ellas, muy habitualmente el primer "componente" de la Agricultura moderna en ser transferido a los agricultores, y que es adoptado por estos últimos, son las semillas mejoradas. No obstante, mientras es posible, estas siguen siendo cultivadas con los métodos y prácticas de cultivo tradicional (manejo y mantención de la fertilidad, manejo de plagas y enfermedades, etc.).

En los sistemas tradicionales el potencial productivo se encontraba determinado y condicionado por la disponibilidad de recursos locales, el grado de eficiencia en la utilización de estos recursos y de la adaptación de los cultivos a estas condiciones. Así, por ejemplo, en el caso de la fertilidad, esta se mantiene en los sistemas mediante la integración de las zonas circundantes al predio y de la producción animal, utilización de técnicas de promoción de la renovación y solubilización natural de nutrientes del suelo, utilización de abonos de origen vegetal o animal y ocasionalmente algunas sales y minerales. Un rasgo propio de la fertilización en la agricultura tradicional, es que los nutrientes aportados por el hombre apenas llegan a cubrir los extraídos por la cosecha, sin que ello redunde en un obligado deterioro de la fertilidad del suelo (Naredo 1996). Ello implica que se debía mantener un equilibrio entre la extracción de nutrientes realizada por las variedades tradicionales y el aporte al sistema. 
La entrada de las nuevas variedades tiene como consecuencia inicial un considerable incremento de los rendimientos, lo cual se debe principalmente a que presentan una mayor capacidad de utilizar los nutrientes solubles y de transformar estos últimos en rendimiento. Así también, esta mayor productividad resulta ser un incentivo para que otros agricultores incorporen las variedades mejoradas. Cuando esta incorporación se ha realizado sin acompañarse de la aplicación de fertilizantes químico-sintéticos el incremento de los rendimientos ha obedecido por lo general a la mayor capacidad de las "nuevas variedades" en extraer rápidamente los nutrientes acumulados por el sistema. Podría decirse que, en esta situación, el aumento en los rendimientos se produce a costa de los "ahorros" del sistema. Como ejemplo podemos mencionar el caso de las comunidades mapuche-navche del sur de Chile, en las cuales se encontró que la gran mayoría de los agricultores ha tenido acceso a estas semillas por medio de intercambios con otros comuneros o venta por parte de los agricultores más tecnificados. Dado que estos no disponen de los recursos para la compra de insumos y que su conocimiento agrícola se basa en las prácticas tradicionales, por lo general comienzan a cultivar estas variedades confiando en sus prácticas de mantención de la fertilidad y muchas veces en la "fuerza de la tierra". Al igual que lo que ocurre en el caso de los mapuche, múltiples estudios desarrollados en distintas zonas de Latinoamérica, Asia y Africa, han mostrado como los primeros años tras la adopción de las nuevas semillas los rendimientos son incrementados significativamente, 10 cual produce que su utilización se extienda rápidamente (Chrispeels y Sadava, 1994; Evans, 1993; Grigg, 1982; Hobbelik, 1992). Sin embargo transcurridos algunos ciclos anuales la situación cambia dramáticamente y se llega a niveles productivos bastante inferiores a los de partida. Las causas y el tiempo en el cual se produce este efecto es variable y depende de las características de cada sistema y del cultivo que se trate, pero sin duda uno de los principales factores de ocurrencia se debe a que los "ahorros" en fertilidad del sistema son finitos y la ruptura del equilibrio mantenido entre extracción-incorporación de nutrientes (mayor extracción) produce su pérdida.

Pese a los rendimientos más bien modestos que se obtienen con las variedades tradicionales, estas y su adaptación a las características locales han permitido que durante siglos los agricultores satisfagan sus necesidades de alimentación y mantenimiento. Como ya fue mencionado, el cambio de estas variedades por las "mejoradas" produce que en un mediano plazo estos sistemas colapsen haciendo necesario la incorporación de fertilizantes comerciales, $\sin$ los cuales muchas veces no se puede pensar siquiera en obtener producción. Junto con el aumento de costos y la necesidad de poseer los recursos monetarios que esto implica, el uso de fertilizantes altamente solubles (como son la mayor parte de los fertilizantes comerciales), especialmente nitrogenados, ocasiona también otra serie de efectos, dentro de los cuales encontramos aumento de la susceptibilidad de las plantas a la incidencia de plagas y enfermedades, aumento de la competencia de las malezas e inhibición de la fauna y microfauna edáfica, entre otros (Altieri, 1999; Altieri, Schmidt y Montalba, 1998; Boeringa, 1980; Chaboussou, 1976; Howard, 1921, 1943, 1947; Hylton, 1973; Hunter, 1971; Kajimuro et al., 1995; Oelhaf, 1978; Phelon y Mason, 1995).

Una de las características comunes de la agricultura tradicional de los variados lugares del planeta (por razones culturales, alimentarias, etc.) la constituye la gran diversidad espacial y temporal presente en sus sistemas, tanto de cultivos, y variedades de estos, como de otros tipos de especies con usos múltiples (medicinales, ornamentales, etc.). Un hecho ya muy estudiado es el que esta diversidad confiere una serie de propiedades benéficas, encontrándose dentro de estas la reducción de la incidencia y daño de las plagas, enfermedades y malezas (Altieri, 1987; Altieri, 1999; Altieri y Whitcomb, 1979; Altieri y Letourneau, 1982; Altieri, Schmïdt y Montalba, 1998; Contreras y Montalba, 1998; De Bach, 1974; Forman y Gordon, 1986; Montalba, 1998; Root, 1973; Wratten y van Emden, 1995).

Al desarrollarse para responder en rendimiento, la aplicación de altas dosis de fertilizantes solubles y de facilitar la mecanización de la producción, las nuevas variedades resultan ser de alturas más bien reducidas y poco competidoras con las malezas. Esta característica, sumada al efecto que la fertilización soluble tiene como promotora del crecimiento de plantas oportunistas, hace imprescindible la utilización de productos herbicidas, los cuales solo pueden ser aplicados en condiciones de cultivos puros. De esta forma, al adoptarse las nuevas semillas y ser reemplazadas las tradicionales, se reduce enormemente la diversidad del sistema y los beneficios asociados a esta.

Considerando las características antes mencionadas, no es de extrañar el hecho de que tras la adopción de las semillas mejoradas deban ser utilizados una serie de insumos petroquímicos (herbicidas, fungicidas, fertilizantes, etc.), sin los cuales la obtención de rendimientos suficientes para la mantención del grupo familiar resulta casi imposible. De esta forma, la productividad de los sistemas familiares pasa a depender mas bien de la disponibilidad de insumos y de su compra en el mercado que de la habilidad de utilización de los recursos prediales y de la mantención de equilibrios al interior del sistema. Cabe destacar que la "adicción" a los agroquímicos en la que incurren los sistemas familiares al adoptar las prácticas modernas, corresponde a un síndrome que afecta al sistema en su conjunto y que se inicia al ser abandonadas prácticas culturales adecuadas, creadas y adaptadas para las condiciones locales, y reemplazadas por un conjunto de prácticas genéricas basadas en insumos externos que deben ser adquiridos en el mercado. De esta forma, resulta ser un gran error el considerar que mediante la realización de ensayos reduccionistas (muy comunes en el desarrollo de la agricultura moderna), como puede ser una simple comparación de "variedades tradicionales" versus "variedades mejoradas" sometiéndolas a distintas dosis de fertilizantes y situaciones de control de malezas (como el desarrollado por Mellado, 1993), pueda ser probada la superioridad de las nuevas variedades para las condiciones "campesinas". 
En los casos en los cuales, gracias al adecuado apoyo de programas de transferencia y financiamiento (subsidio o crédito), los agricultores han adoptado todo el paquete tecnológico y han sido "adiestrados" en su utilización, los procesos descritos anteriormente se aceleran y radicalizan, produciéndose una dependencia acelerada a los insumos y a los créditos (subsidios) necesarios para su compra. Este tipo de adopción, sin embargo, posee la ventaja de presentar un período inicial de bonanzas en el cual el aumento de los rendimientos, producto de la transformación, permite tener excedentes que pueden ser comercializados y generar ingresos que mejoran el nivel de vida de los agricultores y sus familias. De esta forma los casos más "exitosos" pueden, y deben, hacer una completa reestructuración de sus objetivos productivos, debiendo estos orientarse a la obtención de altas producciones para el mercado. Es precisamente en esta etapa de "bonanza" cuando estos agricultores "exitosos" son utilizados como ejemplo a seguir por el resto de los "campesinos".

Lamentablemente, por lo general, al transcurrir algún tiempo ocurren dos procesos que cambian completamente esta situación. Por una parte se comienzan a notar los efectos de la extrema artificialización de los sistemas y de la degradación que en su base de recursos produce el empleo de las nuevas prácticas, reflejándose esto en una mantenida reducción de la producción que para ser recuperada requiere cada vez mayor utilización de insumos (especialmente fertilizantes y pesticidas). En adición a esta reducción de la productividad, la menor estabilidad y capacidad de recobrarse de perturbaciones ambientales que poseen estos sistemas los hace aumentar enormemente los riesgos productivos, los cuales sumados a la inestabilidad que presentan los mercados agropecuarios (en cuanto a oferta y precios) hace que la probabilidad de obtención de pérdidas en una temporada sean muy altos. En el caso de agricultores empresariales estas perdidas pueden ser asumidas (dentro de ciertos límites) e intentar recuperarse en el futuro sin que tenga mayores efectos en la supervivencia del sistema. Para los agricultores familiares, en cambio, esto puede implicar un colapso total del sistema, empobrecimiento, endeudamiento e incluso hambre. Esta situación es mucho más compleja y grave cuando ocurre posterior a finalizado los programas de transferencia y de ayudas gubernamentales, siendo, por lo general, la situación más común.

Por otro lado, una de las características fundamentales de la agricultura moderna, en el marco del sistema capitalista de producción, ha sido atribuida a su tendencia y necesidad de incorporar continuamente innovaciones tecnológicas al proceso productivo, con objeto de reducir los costos de producción y, por consiguiente, aumentar los márgenes de rentabilidad económica. Dentro de este proceso de "espiral tecnológica", la utilización de las nuevas tecnologías por innovadores y adoptantes tempranos suele originar un aumento de la productividad y de la producción agregada de una determinada mercancía beneficiada por el progreso técnico, mientras el precio de dicha mercancía tiende a declinar desproporcionadamente debido a la baja elasticidad de la demanda que suelen presentar los produc- tos agrícolas. El precio relativo del producto cae en la medida en que una técnica tras otra es adoptada en las explotaciones, pero los costos de producción no se reducen (Cochrane, 1964). Para el caso de los agricultores familiares, con escasos recursos e incapaces de seguir la competencia tecnológica, esto se convierte en una pesadilla que hace disminuir cada vez más sus ingresos, encontrándose cada vez más "estrujados" y empobrecidos.

En adición a los efectos "productivos" propiamente tales, la adopción de la «agricultura moderna» en amplias zonas del globo, tanto en países "desarrollados" como en los "sub desarrollados" (y especialmente en estos últimos), ha producido también una serie de problemas cuyo impacto no solo afecta a los sistemas agrícolas en los cuales se ha implementado, sino que trasciende a ellos. Uno de estos efectos negativos lo constituye el hecho de que, pese a que en los sistemas tradicionales la diversidad agraria se había incrementado hasta fechas relativamente recientes (Guzmám, González de Molina y Sevilla, 2000), la incorporación de las nuevas variedades a los diversos países y la adopción de estas por parte de los agricultores, ha desplazado a las variedades (o razas) tradicionales. Es así como entre las bastas consecuencias ambientales y sociales de esta revolución, podemos encontrar un fuerte proceso de uniformidad y erosión genética. Como muestra de esto podemos considerar el hecho de que en la actualidad seis híbridos de maíz conforman más del 70\% del cultivo mundial de esta especie (Gliesman, 1997). De unas 330.000 variedades diferenciadas de arroz que se cultivaban hace 50 años en la india, en 1987 se proyectaba que para el año 2000 solo quedarían 50 (RAFI, 1987). Una sola variedad de arroz (llamada IR-36) se extiende sobre más del $60 \%$ de las tierras arroceras del Sudeste de Asia donde, hace tan solo unos pocos años, eran comunes millares de variedades tradicionales. En oriente medio, las cebadas "Beecher" y "OP25" han acaparado más de la mitad de la cosecha, mientras que el Mexipak y el Sonalika han destruido el caleidoscopio de los trigos y suponen el 70\% de la cosecha (RAFI, 1997). Dada la enorme y rápida erosión genética, producto de la incorporación cada vez más amplia y extensa de los sistemas agrícolas "modernos" y la homogeneización cultural promovida por los organismos de desarrollo, los sistemas agrícolas tradicionales (tanto campesinos como indígenas) presentes principalmente en países del tercer mundo, se han convertido en un gran banco de biodiversidad, la cual es muy requerida al momento de buscar nuevas características en plantas cultivadas que solucionen problemas de la agricultura moderna o que proporcionen características deseadas en "nuevas variedades". Pese a que la agricultura industrializada mundial depende de la diversidad genética que mayoritariamente se ha desarrollado y conservado en sistemas agrícolas tradicionales (presentes mayormente en países del "tercer mundo"), debemos comprender que las variedades tradicionales forman parte del sistema agrícola en su conjunto. Están entretejidas con diferentes prácticas de cultivo, culturas humanas, ecología e historias locales. Por tanto, cuando se extingue una variedad tradicional el más perjudicado no es algún distante programa de producción de variedades: es 
la comunidad y la gente que la forma. La simple extinción de una variedad tradicional puede, a veces, afectar tan profundamente un sistema agrícola nativo que el resultado puede ser un desastre económico o incluso el hambre (RAFI, 1987).

Como ya fue mencionado, la utilización de las nuevas variedades y prácticas agrícolas imponen también la necesidad de utilizar altas dosis de agroquímicos. Pese al enorme uso de pesticidas, que tal como señala Edwars (1993) en la actualidad asciende a cerca de 4.4 millones de toneladas al año, de acuerdo a estimaciones de Pimentel (1993) las pérdidas de cultivos causadas por varios grupos de pestes (malezas, enfermedades e insectos) se han mantenido mas o menos constantes en los últimos 50 años. La razón de lo anterior (según el mismo autor) es que se han substituido buenas practicas agrícolas (como las rotaciones) por pesticidas, lo que su la vez hace surgir otros problemas como la aparición de pestes secundarias al eliminar a los predatores de estas con los pesticidas, y el cada vez mayor número de especies que han creado resistencia a estos. Sin embargo, como ya se dijo, la causa no sería solo esa, sino que también intervienen variados componentes de este sistema, los que hacen susceptibles a los cultivos al ataque de plagas y enfermedades, y la competencia de las malezas. En adición a los problemas ocasionados en los propios sistemas productivos, los pesticidas y fertilizantes industriales han sido asociados con múltiples problemas tanto de contaminación del medio ambiente (suelo, aguas superficiales, aguas subterraneas, alimentos), salud humana, daño a flora y fauna silvestre, efectos negativos en otras actividades económicas, etc. (WHO/UNEP, 1989; Lijinsky, 1989; Misch, 1994; Pimentel et. al , 1993). Pese a que el uso de estas prácticas benefician principalmente a los propietarios de sistemas productivos de características industriales y de gran escala, por lo general los principales afectados suelen ser los trabajadores agrícolas y las comunidades circundantes, lo cual indicaría que, además de una inequidad de los beneficios que estos sistemas proveen, se produce una inequidad también al momento de asumir las externalidades negativas (Martinez-Alier y Roca, 2000).

Pese a que se podría pensar que, dado el suficiente capital y tierras aptas, la propuesta de los nuevos sistemas agrícolas desarrollada con la "revolución verde" resulta completamente viable en los países "desarrollados", la permanencia de estos sistemas en los países antes mencionados solo ha sido posible mediante la aplicación de políticas agrícolas, generalmente basadas en subsidios, las cuales han permitido proveer de adecuados ingresos a los agricuitores y alimentos a un precio relativamente bajo para sus habitantes (Dalberg, 1979; Pretty, 1995). P.or otro lado en muchos de estos países la aplicación de subsidios constituye un incentivo para la aplicación de inputs mucho más altos de los que serían económicamente viables sin estos (desde una perspectiva crematística), incrementando aún mas los problemas ambientales y demás externalidades asociadas a este paquete tecnológico. En forma adicional, la sobreproducción de alimentos promovidos por estas políticas crea impor- tantes problemas en el mercado internacional y en muchas ocasiones tiene serios efectos en la sustentabilidad económica de sistemas agrícolas del tercer mundo, ya que la importación de estos productos, a precios muchas veces inferiores a los costos de producción, es un estímulo para que países del tercer mundo sustituyan las producciones locales, realizadas bajo sistemas tradicionales, por importaciones. Un caso simbólico de esta situación lo constituye el maíz y el acuerdo comercial NAFTA entre Estados Unidos y Méjico, en el cual los sistemas agrícolas tradicionales mejicanos se encuentran gravemente amenazados por importaciones de maíz (fuertemente subsidiadas) desde Estados Unidos (Nadal, 2000). Se debe resaltar también el hecho de que las necesidades de subsidios para permitir los niveles de producción propios de estos sistemas no se limitan solamente a los países desarrollados, sino que también una importante parte del ingreso de muchos países en desarrollo se utiliza en subsidiar los insumos requeridos.

De esta forma, y en gran parte a causa de lo expuesto en este trabajo, al ser exportados estos modelos agrícolas a países del "Tercer Mundo", se exacerbaron aún más los problemas ambientales y sociales que había en estos. La mayor parte de los agricultores de escasos recursos de América Latina, Asia y África ganaron muy poco en el proceso de desarrollo y transferencia de tecnología de la Revolución Verde, porque las tecnologías propuestas no fueron neutras en cuanto a escala. Los agricultores con tierras más extensas y mejor mantenidas (a la vez con mayor acceso a créditos y "subsidios") ganaron más, pero los agricultores con menores recursos que viven en ambientes marginales perdieron con mayor frecuencia y la disparidad de los ingresos se vio acentuada (Conway, 1997). El cambio tecnológico ha favorecido principalmente la producción y/o exportación de cultivos comerciales producidos, sobre todo, por el sector de las grandes propiedades, con un impacto marginal en la productividad de los cultivos para la seguridad alimentaria, mayormente en manos del sector "campesino" (Pretty, 1995). En las áreas donde se realizó el cambio progresivo de una agricultura de subsistencia a otra de economía monetaria, se pusieron en evidencia gran cantidad de problemas ecológicos y sociales: pérdida de autosuficiencia alimentaria, erosión genética, perdida de la biodiversidad y del conocimiento tradicional, e incremento de la pobreza rural (Conroy et al, 1996).

Debido a estos y muchos otros factores, es que al cabo de tres décadas de haber sido adoptada a gran escala en el "Tercer Mundo", 1a Revolución Verde no pudo resolver los problemas a los que se enfrentaba. El hambre, que es función no tanto de la producción sino que de la distribución y el consumo, sigue siendo un problema tan grave como cuando las voces esperanzadas de la Revolución Verde proclamaron por primera vez una solución a la crisis de alimentos (BALAI, 1987). Resulta evidente que si bien la nueva tecnología ha aumentado enormemente la productividad en el corto plazo, ha disminuido también la sustentabilidad, la equidad, la estabilidad y la productividad a largo plazo de los sistemas agrícolas. 
Pese a que podríamos seguir analizando los múltiples efectos sociales, culturales, económicos y ambientales que ha implicado la adopción del nuevo modelo agrícola para los sectores de agricultura familiar tradicional (campesinos e indígenas), los antecedentes presentados cuestionan prácticamente a todo nivel, que sea el sistema más adecuado para superar la pobreza en los sectores campesinos e indígenas y mucho más para alimentar al mundo. De hecho lejos de disminuir el hambre tras la revolución verde, esta se ha incrementado (Altieri, 1999).

\section{Conclusiones}

Según se ha podido mostrar en el presente trabajo, la forma en la cual se ha ido desarrollando la agricultura moderna (sometida a los más diversos tipos de presiones e intereses) y los graves efectos de esta tanto en el medio ambiente, la salud humana, las sociedades y culturas, así como en la misma economía de los agricultores y los países del tercer mundo, nos dan claros indicios de que el camino seguido no es el ambientalmente más adecuado, el socialmente más justo, el técnicamente mejor, ni el económicamente mas viable (salvo si se ve desde el punto de vista de las grandes empresas proveedoras de insumos). Se trata en definitiva, de un modelo altamente insostenible en el largo plazo y totalmente inadecuado para la solución de los problemas que enfrentan los sistemas campesinos e indígenas de los países del "tercer mundo".

Pese a las constantes valoraciones positivas que se han hecho de la agricultura tradicional a lo largo de todo este trabajo, no es mi intención el pretender que, en la situación que actualmente enfrentan los campesinos e indígenas de los diversos países, se debiera regresar completamente a este tipo de prácticas, sino que, de igual modo que los sistemas tradicionales, los modelos de agricultura y desarrollo agrícola que se pretendan seguir o aplicar en estos contextos debieran ser mucho más adecuados a las características ambientales, económicas, sociales y culturales de los diversos lugares. Sin embargo, también debo destacar que en muchos lugares del mundo la agricultura tradicional sigue siendo una alternativa completamente valida y la que mayormente consigue estos objetivos, existiendo ejemplos dignos de imitar por otros sistemas agrícolas.

\section{Bibliografía}

ALTIERI, M., (1999). Agroecología. Bases científicas para una agricultura sustentable. Editorial Nordan-Comunidad. Montevideo. Uruguay. 338 pág.

ALTIERI M.A., L. SCHDMIDTH \& R. MONTALBA (1998). Assessing the efects of agroecological soil managenet practices on broccoli insect pest population. En: Biodynamics, julio-agosto 1998.Págs. 23-28.
ALTIERI, M.A. \& WHITCOMB. (1979). The potential use of weeds in the manipulation of beneficial insects. En: Horticulture Science (EE.UU.) 14 : 12 - 18.

BOERINGA, R.(1980). Alternative methods of agriculture. Elsevier, Amsterdan, 200 págs.

CHABOUSSOU, F. (1975). Cultural factors an the resistance of citrus plants to scale insects and mites. En: Fertilizer use and plant health, international Potash Institute, worblaufen-bern, Suecia, págs. 259-280.

CHRISPEELS, M. \& D. SADAVA (1994). Plants, Genes, and agriculture. Jones and Bartlett Publishers. Boston, Estados Unidos. 477 págs.

CONTRERAS, A. \& R. MONTALBA (1998). Agroecologic handing records on pest performed by the mapuche people of Chile. Libro de conferencias XII congreso científico internacional IFOAM. Mar del Plata, Argentina.

CONTRERAS A. M. (1987). Germoplasma chileno de papas (Solanum spp.). En: Anales Simposio Recursos Fitogenéticos. UACH-IBPGR. Valdivia, Chile

CONROY. M.T., D.L. MURRAY \& P. ROSSET (1996). A cautionary fable:failed US development policy in Central America. Boulder. CO: Lynne Rienner Publishers.

CONWAY, G.R. (1997). The doubly Green Revolution: food for all in the 21st century. London, UK: Penguin Books.

COSTABEBER, J.A. (1998). Acción colectiva y proceso de transición agroecológica en Rio Grande do Sul, Brasil. Tesis Doctoral presentada en la Escuela Técnica Superior de Ingenieros Agrónomos y de Montes de la Universidad de Córdoba (España).

DAHLBERG, K. (1979). Beyond the green revolution; the ecology and politics of global agricultural development. Plenum Press, New York. U.S.A., 256 págs.

DE BACH, P. (1974). Biological Control by Natural Enemies., Cambridge University Press, Cambridge, EE.UU., 235 págs.

EDWARS, C.(1993). The impact of pesticides on the environment. En: The pesticide question, environment, economics and ethics. D. Pimentel y H. Lehmen (editores), Chapman \& Hall. New York. USA. Págs 13 - 46.

ESCOBAR, A. (1996). The making and unmaking of the third world. Princeton University Press. 560 págs.

EVANS, L. (1993). Crop, evolution, adaptatión and yield. Cambridge University Press. U.K., 500 págs.

FORMAN, R.T.T. \& M. GORDON (1986). Landscape Ecology. John Wiley and Sons, New York, 619 págs. 
GLIESSMAN, S. (1997). Agroecology. Ecological processes insusteinable agriculture. Ann Arbor Press. Cheise. USA.

GRAIN (2000). Patato: a fragile gift from the Andes. En: Seedling, septiembre 2000, Volumen 17, $\mathrm{n}^{\circ} 3$. Genetic Resources Action International (GRAIN). Pags. 23-35.

GUZMAN, G., M. GONZÁLEZ DE MOLINA \&E. SEVILLA (2000). Introducción a la agroecología como desarrollo rural sustentable. Ediciones Mundi-Prensa, Madrid, España. 534 Págs.

HOWARD, A. (1921). The influence of soil factors on disease resistance: En: Ann. Appli. Biol., 7: 373-389.

HOWARD, A. (1943). An Agricultural Teatament. Oxford University Press, Londres, Inglaterra. 253 págs.

HOWARD, A. (1947). The soil and healt. A Study of Organic Agriculture. Devlin-Adair, Nueva York, EE.UU., 307 págs.

HUNTER, B.T.(1971). Gardening without poisons. Houghton Miffin, Boston, EE.UU. 318 págs.

HYLTON, W.H.(1973). Organically Grown Foods. Rodale Press, Emmaus, Pennsylvania, E.E.U.U. 98 págs.

KAJIMURA T., I.N. WIDIATRA, K. NAGAI, K. FUJISAKI \& F. NAKASUJI (1995). Effect of organic rice farming on Planthoppers, Reproduction of the White Backed Planthopper, Sogatella furcifera Horvath. Respouse. Population Ecology 37 (2): 219-224.

MARTINEZ-ALIER J. \& J. ROCA (2000). Economía ecológica y política ambiental. PNUMA, Fondo de Cultura Económica. Méjico. 493 págs.

MELLADO, M. (1993). Mejoramiento de trigo y sustentabiliḍad. Contribución del mejoramiento genético de trigo para una agricultura sustentable. IPA Quilamapu 57:3 - 6 .

MISCH, A. (1994). Riesgos ambientales para la salud: una evaluación. En: La situación del mundo 1994, Brown, L. R. (coordinador). Emecé Editores \& CIP/FUHEM. Barcelona, España.

MONTALBA, R. (1998). Diversificación de agroecosistemas y fertilización orgánica; una aproximación agroecológica al manejo de plagas. Tesis de grado presentada en la Facultad de Ciencias Agropecuarias y Forestales de la Universidad de La Frontera, como parte de los requisitos para optar al título de ingeniero agrónomo. Temuco, Chile.

NADAL, A., 2000. El caso del maíz mejicano en el NAFTA; variabilidad genética y liberalización comercial. En: Biodiversidad, Sustento y Culturas, $n^{\circ} 24$, julio 2000. Pags. 312.
NAREDO, J. M., 1996. Sobre la reposición natural y artificial de agua y de nutrientes en los sistemas agrarios y las dificultades que comporta su medición y seguimiento. En: La fertilización en los sistemas agrarios, una perspectiva histórica. $\mathrm{R}$ Garrabou y J. M. Naredo (editores). Fundación Argentaria. Madrid, España. Págs.17-34.

OELHAF, R.C., 1978. Organic agriculture: Economic and Ecological Comparisons with conventional methods. wiley, Nueva York. EE.UU., 271 págs.

PHELAN P.L., MASON J.F. AND STINNER B.R., 1995. Soilfertility management and host preference by Europian com borer, Ostrinia nubilaris (Hubner), on Zea mays L.: A comparison of organic and conventional chemical farming. Agriculture: En: Ecosistems and Enviroment, $\mathrm{n}^{\circ} 56$. págs. 1-8.

PIMENTEL, D., H. ACQUAY, M BLTONEN. P. RICE, M. SILVA, J. NELSON, V. LIPNER, S. GIORDANO, A. HOROWITZ Y M. D AMORE. 1993. Assessment of environmental and economic impacts of pesticides. En: The pesticide question, environment, economics and ethics. D. Pimentel y H. Lehman (editores). Chapman \& Hall. New York. USA. Págs 47 - 84.

PRETTY, J., 1995. Regenerating agriculture: policies andpractices for sustainability and self-reliance. London, U.K.: Earthscan.

PUJOL, J., 1998. Las innovaciones biológicas en la agricultura española antes de 1936; el caso del trigo. En: Agricultura y Sociedad, $\mathrm{n}^{\circ} 86$ (mayo-agosto 1998), Pags. 163-162.

RAFI, 1987. ¿De donde vienen las semillas....y adonde van?. En: Mas allá de la Revolución Verde; Las nuevas tecnologías genéticas para la agricultura, ¿desafío o desastre?. Henk Hobbelink (Ed.). Editorial Lerna S.A., Barcelona, España, Pags. 13-33.

ROSS, E.B., 1998. The Mualtus Factor. Population, poverty and politics in capitalist development. Zed Books Ltda, Londres, Inglaterra. 257 págs.

ROOT, R.B., 1973. Organization of a plant-anthropod association in simple and diverse habitats: the fauna of collard (Brassica oleracea). En: Ecological. Monography, $n^{\circ} 43$ págs. 95-124.

SEVILLA GUZMÁN E. Y M. GONZALEZ DE MOLINA, 1990. Ecosociología: elementos teóricos para el análisis de la coevolución social y ecológica. En: Revista Española de Investigaciones Sociológicas, $\mathrm{n}^{\circ} 52$, págs 7 - 45 .

SEVILLA GUZMÁN, E. Y A. LÓPEZ CALVO, 1994. Agroecología y campesinado. En: Agriculturas mediterráneas y mundo campesino. A. Sánchez Picón (editor). Instituto de Estudios Almerienses. Diputación de Almería. Págs. 69-92. 
TOLEDO, V. M., 1993. La racionalidad ecológica de la producción campesina. En: Ecología, campesinado e historia. E. Sevilla Guzmán y M. González de Molina (editores). La Piqueta. Madrid, España. Págs. 197-218.

WHO/UNEP, 1989. Public healt impact of pesticide used in agriculture. Geneva: World Healt Organization/United Nations Environment Programme.

WRATTEN, S.D. Y H.F. VAN EMDEN, 1995. Habitat management for enhanced activity of natural enemies of insect pests. En: Ecology and Integrated Farming Systems; D.M. Glen, M.P. Greaves, y H.M. Anderson, editores. Bristol: John Wiley y Sons Ltd. EE.UU., 125 págs. 